\title{
ENERGY TRANSFER IN TRIMERIC C-PHYCOCYANIN STUDIED BY PICOSECOND FLUORESCENCE KINETICS
}

\author{
J. Wendler ${ }^{1}$, W. John ${ }^{2}$, H. SCheer ${ }^{2}$ and A. R. Holzwarth ${ }^{1 *}$ \\ 'Max-Planck-Institut für Strahlenchemie, Stiftstr. 34-36. D-4330 Mülhcim a.d. Ruhr and \\ 'Botanisches Institut der Universität, Menzingerstr. 67, D-8000) München 19, W. Germany
}

(Received 22 January 1986; accepted 6 February 1986)

\begin{abstract}
The excited state kinetics of trimeric C-phycocyanin from Mastigocladus laminosus has been measured as a function of the emission and excitation wavelength by the single-photon timing technique with picosecond resolution and simultaneous data analysis. A fast decay component of 22 ps (C-phycocyanin with linker peptides) and $36 \mathrm{ps}$ (C-phycocyanin lacking linker peptides) is attributed to efficient energy transfer from sensitizing to fluorescing chromophores. At long detection wavelengths the fast decay components are found to turn into a rise term. This finding further corroborates the concept of intramolecular energy transfer. Previous reports on the conformational heterogeneity of the chromophores and/or proteins in C-phycocyanin are confirmed. Our data also provide indications for the importance of the uncoloured linker peptides for this heterogeneity.
\end{abstract}

\section{INTRODUCTION}

C-Phycocyanin is the major light harvesting pigment of many cyanobacteria (blue-green algae). In situ, it is highly aggregated and organized together with other phycobiliproteins and colorless linker peptides in the supramolecular complexes called phycobilisomes. They constitute the antenna to (mainly) photosystem II (Gantt, 1981; Scheer, 1982; Glazer, 1983; Wehrmeyer, 1983a; Wehrmeyer, 1983b). In vitro, in particular in the absence of linker peptides, the aggregation is limited to trimers (Hefferle et al., 1984a) or hexamers (see MacColl and Berns, 1981, for leading references) which are believed to be the building blocks for phycobilisomes (Wehrmeyer, 1983a; Glazer, 1983; Gantt, 1980) and phycocyanin crystals (Schirmer et al., 1985a; Schirmer et al. . 1985b)

The phycobilisomes collect efficiently light in the green and orange spectral range and transfer it with quantum efficiencies approaching $100 \%$ to the reaction centers (Porter et al., 1978). Several groups have recently begun to study the kinetics of this process by picosecond time-resolved spectroscopy of entire phycobilisomes and their constituent phycobiliproteins (Hefferle et al., 1983a; Hefferle et al., 1983b; Porter et al., 1978; Searle et al., 1978; Kobayashi et al., 1979; Pellegrino et al., 1981; Holzwarth et al., 1982; Holzwarth et al., 1983b; Suter et al., 1984; Hefferle et al., 1984a; Hefferle et al., 1984b; Gillbro et al., 1983; Mimuro et al., 1984; Yamazaki et al., 1984; Switalski and Sauer, 1984; Wong et al., 1981; Holzwarth, 1985; Wehrmeyer et al., 1985; Gillbro et al., 1985; Mimuro et al., 1985; Glazer et al., 1985; Hanzlik et al., 1985; for recent reviews see Karukstis and Sauer, 1983; Scheer, 1985). These studies support a general scheme, where the excitation energy

\footnotetext{
*To whom correspondence should be addressed.
}

flows from the highest energy components (generally the phycoerythrins) via the intermediate phycocyanins to the lowest energy components, e.g. the allophycocyanins and in vivo to the chlorophylls. From these studies there remained nonetheless considerable differences regarding the laws governing the deactivation kinetics (multi-exponential or nonexponential), the number of components, and the physical interpretation of the derived time constants. These differences are, at least in part, due to the application of experimental techniques differing in their time resolutions and their capabilities for multicomponent resolution. Further differences derive from pigment systems differing in their parent species, aggregation states, and preparation procedures. In order to correlate the data obtained in different laboratories, and to compare critically the potential of the experimental methods, parallel investigations using different techniques are therefore useful. Here, we wish to report the fluorescence kinetics of trimeric C-phycocyanin from Mastigocladus laminosus measured by the single-photon timing technique. We also apply for the first time the simultaneous data analysis technique to such a complex pigment system with energy transfer. This technique drastically improves the capability for multicomponent resolution of complex decay data. The results will be compared with polarized fluorescence data obtained previously on the same pigment with a repetitive streak camera (Hefferle et al., 1984a). C-Phycocyanin from this alga has been characterized earlier using biochemical methods by Binder et al. (1972) and Byfield and Zuber (1972).

\section{MATERIALS AND METHODS}

C-Phycocyanin was isolated from Mastigocladus laminosus as reported carlier (Hefferle et al. 1984a). One of the preparations was $\geq 80 \%$ pure with respect to the $C$ phycocyanin biliprotein and contained colorless peptides as 
analyzed by SDS-PAGE ( $c f$. Fig. 1, lane b). Two of these polypeptides (22.2 and $25.4 \mathrm{kDa})$ are associated with phycocyanin in the phycobilisomes and are also isolated with phycocyanin upon dissociation of the phycobilisomes (unpublished results, Scheer, 1985). The relative staining intensities of $\alpha+\beta$, the 22.2 and $25.4 \mathrm{kDa}$ subunits are $25: 1: 2$. All other peptides $(>26 \mathrm{kDa})$ are contaminants due to the isolation procedure and are not found in phycobilisomes. The other preparation was freed from polypeptides other than the two C-phycocyanin subunits as shown in Fig. 1 (lane c). All samples were prepared in potassium phosphate buffer $(80 \mathrm{mM}, \mathrm{pH} 6.0)$ containing sodium azide $(3.0 \mathrm{~m} M)$. They were kept on ice until the measurement which was carried out within $48 \mathrm{~h}$. For the measurements the sample was diluted to a chromophore concentration of $<5 \times 10^{-6} M$ corresponding to an absorbance $A_{620}^{1} \mathrm{~cm}<0.5$. For fluorescence measurements a pathlength of $1.5 \mathrm{~mm}$ was used in order to avoid selfabsorption effects. The aggregation state of these solutions was determined to be trimeric (sedimentation constant $S_{20, w}=$ 5.6) for both preparations by analytical ultracentrifugation (see Hefferle et al., 1984a).

Picosecond fluorescence decays have been measured by the single-photon counting technique as described (Holzwarth et al., 1982; Holzwarth et al., 1983a; Holzwarth et al., $1983 \mathrm{~b}$ ). The apparatus function was $\leqslant 120 \mathrm{ps}$ wide (fullwidth at half maximum). This system provides a timeresolution of about $10 \mathrm{ps}$ by deconvolution. The detection wavelength range was selected by a monochromator (Jobin Yvon DH 10) with a bandwidth of $4 \mathrm{~nm}$. All measurements have been carried out at ambient temperature $\left(22-25^{\circ} \mathrm{C}\right)$ under magic angle polarization, i.e. the fluorescence kinetics free from anisotropy effects has been detected.

\section{DATA ANALYSIS}

The data analysis has been carried out using two different methods. The first one involves the more conventional deconvolution of single fluorescence decays in terms of a sum-of-exponentials model (Holzwarth et al., 1982; Holzwarth et al., 1983b). The second method is a simultaneous analysis procedure based on a global optimization algorithm similar to the one used by Knutson et al. (1983) (Holzwarth et al., to be published). Instead of analyzing individual decay curves recorded at one wavelength, all decay curves recorded at different wavelengths are analyzed simultaneously in a single run. The algorithm is based on the assumption that the decay constant (lifetime) of a particular decay component should be independent of wavelength while the preexponential factors vary. The fluorescence decay is described by a sum of exponential functions

$$
F(t, \lambda)=\sum_{\mathrm{i}=1}^{\mathrm{n}} A_{\mathrm{i}}(\lambda) \exp \left(-t / \tau_{\mathrm{i}}\right)
$$

If the lifetimes are independent of emission wavelength the dimensionality of the fitting problem can be reduced from $2 \times N \times n$, to $N \times n+n$ by applying the global analysis. Here, $N$ denotes the number of independent measurements at different wavelengths and $n$ the number of decay components. We have recently derived analytical expressions for the improvement in the errors of the extracted parameters as compared to an independent analysis of the individual decay curves (to be published). The

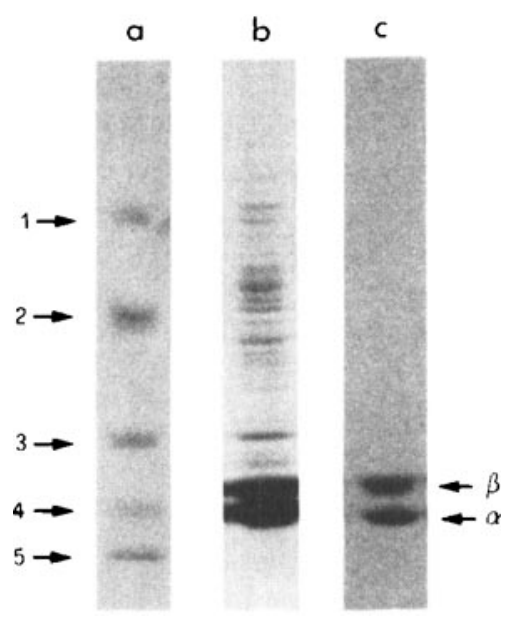

Figure 1. SDS polyacrylamide gel electrophoresis of (a) standard proteins ( 1 bovine serum albumin $\mathrm{mol} \mathrm{wt}=66$ $\mathrm{kDa}, 2$ egg albumin mol wt $=45 \mathrm{kDa}, 3$ trypsinogen mol wt $=24 \mathrm{kDa}, 4 \beta$-lactoglobulin mol wt $=18.4 \mathrm{kDa}$ and 5 Lysozyme mol wt $=14.3 \mathrm{kDa}$ ), (b) phycocyanin preparation containing additional polypeptides (gel overloaded to show the latter more clearly) and (c) phycocyanin preparation without such polypeptides. The phycocyanin subunits are marked $\alpha$ and $\beta$.

reduction in the dimensionality of the free parameters leads to a dramatic improvement both in the accuracy of the extracted parameters as well as in the capability for multi-component resolution. The quality of the fits was judged by a global $\chi^{2}$-value, individual $\chi^{2}$-values, and plots of the weighted residuals. The iteration procedure applied in our program is a semi-linear Marquardt algorithm (Marquardt, 1963). The feasibility and reliability of the four-exponential analysis has been tested on a number of simulated data sets with three and four decay components, which mimicked the experimental data. Poissonian noise had been added to these simulated decay data. These tests confirmed the expected drastic improvement of the global data analysis as compared to the conventional single-decay analysis. In all these simulations the theoretical parameters (amplitudes and lifetimes) were recovered very closely which makes us confident that the present analysis is both reliable and accurate.

\section{RESULTS AND DISCUSSION}

All measurements were carried out on the two different C-phycocyanin preparations. Both were trimeric as determined by ultracentrifugation, but differed by the presence and absence, respectively, of additional polypeptides other than the $\alpha$ - and $\beta$ subunits of $\mathrm{C}$-phycocyanin. Of these additional polypeptides, two are isolated as integral components of the phycobilisomes, from which they dissociate together with $\mathrm{C}$-phycocyanin upon incubation with buffers of decreased ionic strength (John and Scheer, unpublished results). These two 
polypeptides (22.2 and $25.4 \mathrm{kDa}$, see experimental part) are therefore considered as linker peptides, which have been characterized in detail for other species (see Glazer et al., 1983, for leading references). In $M$. laminosus phycobilisomes, the detailed function and the stoichiometry of the different linker polypeptides is still unclear. If judged from the relative staining intensities of the bands (see experimental part) and the trimeric nature of the C-phycocyanin, a heterogeneous population of trimers in the sample which contained different linkers is likely.

Fluorescence decays of both trimeric C. phycocyanin preparations have been measured at various excitation/emission wavelength pairs. At wavelengths $\lambda_{\mathrm{cxc}} \leqslant 605 \mathrm{~nm}$ the s-chromophores should be preferentially excited, whereas at $\approx 620$ $\mathrm{nm}$ the f-chromophores t contribute the largest part to the absorption (Teale and Dale, 1970; Glazer et al., 1973; Switalski and Sauer, 1984). The residual plots resulting from a simultaneous analysis of the fluorescence decays from the preparation that included the linker peptides are shown in Fig. 2. Threeand four-exponential model functions have been used for the analysis presented in Figs. $2 a$ and $2 b$, respectively. These data clearly indicate the requirement for a four-exponential model to describe the fluorescence decays. By the same criteria the preparation that did not contain linker peptides required also four components but the amplitude ratios and lifetimes were different. All lifetimes and amplitudes for the decay components of the two phycocyanin preparations are presented in Table 1 .

\section{Short-lived fluorescence components}

In both preparations we observe a very short-lived fluorescence decay, $\tau_{1}=22$ and $36 \mathrm{ps}$, respectively, at short excitation and emission wavelengths. Its ampli-
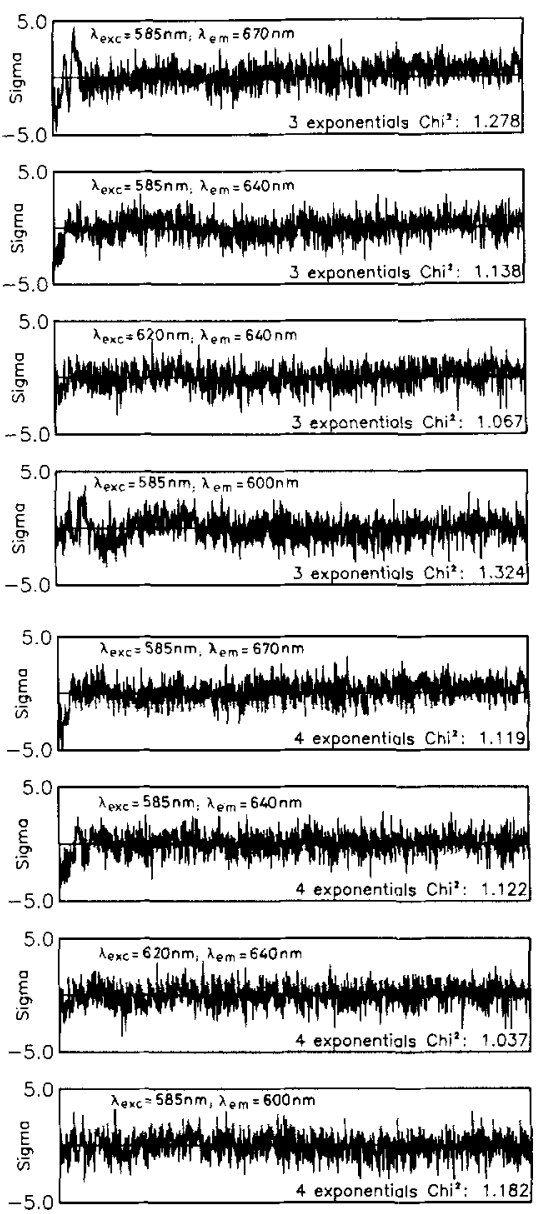

Figure 2. Residual plots resulting from a simultaneous analysis of the fluorescence decavs of trimeric Cphycocyanin from Mastigocladus laminosus including linker peptides. Three-exponential (a) and four-exponential (b) model functions have been applied (for the kinetic data see Table 1). Note the systematic deviations in the residuals of the three-exponential analysis. The decays have been analyzed over a time range of $\approx 10 \mathrm{~ns}(c f$. Fig. 3$)$

Table 1. Lifetimes $(\tau)$ and amplitudes (A) of the fluorescence decay components from trimeric C-phycocyanin as analyzed by the simultaneous deconvolution procedure

\begin{tabular}{|c|c|c|c|c|c|c|c|c|c|}
\hline $\begin{array}{c}\lambda_{\mathrm{cxc}} \\
(\mathrm{nm})\end{array}$ & $\begin{array}{c}\lambda_{\mathrm{em}} \\
(\mathrm{nm})\end{array}$ & $A_{1}{ }^{*}$ & $\mathrm{~A}_{2}{ }^{*}$ & $A_{3}{ }^{*}$ & $A_{4}^{*}$ & $\tau_{1}^{\dagger}$ & $\tau_{2}^{\dagger} \uparrow$ & $\tau_{3} \dagger$ & $\tau_{t^{\dagger}}^{\dagger}$ \\
\hline $585 \neq$ & 600 & 1 & 0.087 & 0.15 & 0.21 & & & & \\
\hline $585 \ddagger$ & 640 & -0.017 & 0.043 & 0.18 & 0.24 & 36 & 203 & 807 & 1420 \\
\hline $620 \ddagger$ & 640 & -0.15 & 0.075 & 0.24 & 0.33 & & & & \\
\hline $585^{\S}$ & 600 & 1 & 0.12 & 0.13 & 0.14 & & & & \\
\hline $585^{\S}$ & 640 & -0.43 & 0.063 & 0.16 & 0.20 & 22 & 120 & 800 & 1490 \\
\hline $620^{8}$ & 640 & -0.086 & 0.026 & 0.095 & 0.12 & & & & \\
\hline
\end{tabular}

*All amplitudes have been normalized to the largest decay component of each sample; a negative amplitude denotes a rise-term. Absolute amplitudes can not be deduced from these data because different neutral density filters have been used for the measurements at different wavelength pairs in order to compensate for the largely differing fluorescence intensities.

+ All lifetimes in ps; the maximum errors for amplitudes and lifetimes are $\pm 10 \%$.

$\ddagger$ C-phycocyanin without linker peptides.

${ }^{8}$-phycocyanin including linker peptides.

$\downarrow$ Abbreviations: f-chromophore(s), fluorescing chromophore(s); s-chromophore(s), sensitizing chromophore(s). 
tude is very prominent under these conditions (see Table 1). The short-lived decay turns into a fluorescence rise-term (negative amplitude) in both preparations when the fluorescence is detected at or above $640 \mathrm{~nm}$. This finding provides direct evidence in favor of ultrafast energy transfer between chromophores whose absorption and emission spectra are shifted against each other, i.e., $s \rightarrow f$ transfer. A similarly fast fluorescence decay also turning into a rise-time at long wavelengths, has been observed recently with B-phycoerythrin aggregates (Wehrmeyer et al., 1985) and phycocyanin 612 (Hanzlik et al., 1985). We should like to note that the basic features of the fluorescence decay of C-phycocyanin, i.e. a fast decay at short wavelength and a rise-term at long wavelength, appear already in a conventional single decay analysis. However, the statistical errors in both lifetimes and the amplitudes are much reduced by the simultaneous analysis and the distinction between three and four components is much more pronounced. An example of a single decay analysis is provided in Fig. 3. This example shows that a conventional analysis of a decay recorded at one wavelength is not able to resolve the details of the fast energy transfer components in this case.

Previous picosecond studies on isolated phycobiliproteins revealed only fast decay components without rise-terms (Kobayashi et al., 1979; Holzwarth et

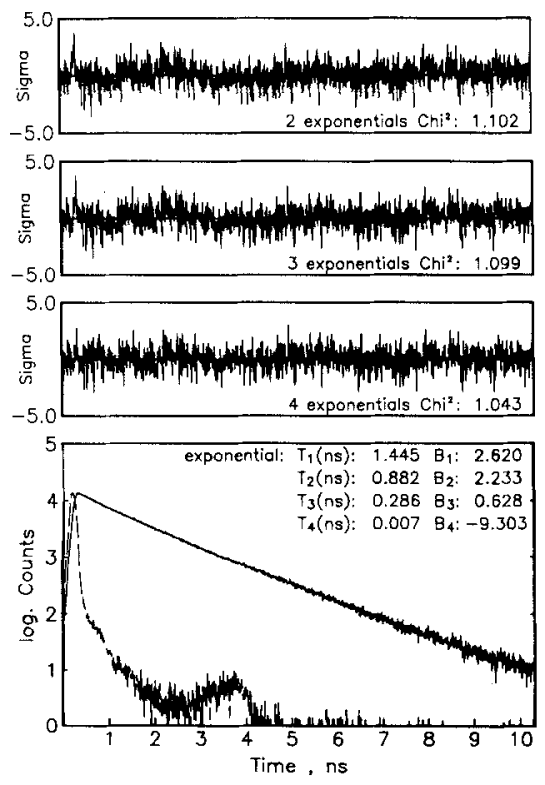

Figure 3. Conventional single decay analysis of the fluorescence kinetics of $\mathrm{C}$-phycocyanin including linker peptides; $\lambda_{\text {exc }}=585 \mathrm{~nm}, \lambda_{\mathrm{em}}=640 \mathrm{~nm}$. Lawer panel: Excitation function (dashed) and fluorescence decay (full line) on a semilogarithmic scale. The inset gives the lifetimes $\left(T_{i}\right)$ and the absolute amplitudes $\left(B_{i}\right)$. Note the negative amplitude $B_{4}$ which indicates a riseterm in the fluorescence kinetics. Upper panels: Weighted residuals plots for model functions of two, three, and four exponentials, respectively. The different kinetic models are hardly distinguishable in this single decay analysis in contrast to the simultaneous analysis (Fig. 2). al., 1983b; Pellegrino et al., 1981) which were interpreted in terms of $s \rightarrow f$ energy transfer (Kobayashi et al., 1979; Holzwarth et al., 1983b) or exciton annihilation (Pellegrino et al., 1981). The latter complication can be definitely excluded for the data presented here since we worked at photon densities well below $10^{11}$ photons $/ \mathrm{cm}^{2} /$ pulse. It shoud be pointed out that the observation of a corresponding risetime component for the fast decays constitutes a prerequisite for an unequivocal assignment of the fast $\left(\tau_{1}\right)$ fluorescence component to intramolecular energy transfer. This result is in excellent agreement with the observation of similarly fast decay components of fluorescence, transient absorption, and transient absorption anisotropy in the C-phycocyanin rods of phycobilisomes from Synechococcus 6301 (Suter et al., 1984; Gillbro et al., 1983; Gillbro et al., 1985) and in isolated C-phycocyanin aggregates (Holzwarth, 1985) from the same cyanobacterium.

The very short isotropic decay component found here by single-photon timing should be compared to the fast fluorescence anisotropy decay measured recently on the same pigment with a synchronous streak camera (Hefferle et al., 1984a) (fast component of the difference function $\approx 62 \mathrm{ps}$, depolarization time estimated to $\approx 70 \mathrm{ps}$ ) which has been attributed mainly to $s \rightarrow f$ energy transfer. Taking into account the lower signal/noise ratio and lower dynamic range of the streak camera, which allowed only analysis in terms of a double-exponential model, it can be concluded, that the fluorescence anisotropy decay (Hefferle et al. 1984a) is in agreement with the results reported here. The fast decay and rise components had not been observed in the isotropic decay, however. This might be due to the fact that broadband filters had been used to select the fluorescence in contrast to the narrow band detection $(4 \mathrm{~nm}$ bandwidth) used in this work. A similar conclusion applies to the picosecond absorption measurements of Kobayashi et al. (1979). Given the limited capability for multicomponent resolution of that method, their results $(\tau \approx 56$ ps for trimeric aggregation) agree favorably well with ours. It should be noted, however, that their C-phycocyanin had been isolated from a different organism. Due to the overlap of the spectra of ' $s$ '- and ' $f$ '-chromophores the relative amplitude of the fast components at $\lambda_{\mathrm{em}}=640 \mathrm{~nm}$ depends strongly on the excitation wavelength. This indicates that this wavelength $\left(\lambda_{\mathrm{em}}=640 \mathrm{~nm}\right)$ is close to the wavelength where the zero-crossing occurs, i.e., the amplitudes of the fast decay term and the fast riseterm compensate. We estimate that this zerocrossing occurs in the wavelength range between 630 and $640 \mathrm{~nm}$. A similar observation has been made for phycocyanin aggregates from Synechococcus 6301 (Holzwarth, 1985). This behaviour provides a further explanation for the failure of some previous investigators to detect the isotropic fast component. If broadband detection is used, the amplitude of the fast component will always be reduced to a varying 
extent due to compensation effects. In unfavourable cases this component could thus be missed completely. Similarly the fast component will not be detectable if narrow-band detection near the zero-crossing wavelength is used as the only detection wavelength. We should like to point out that in all the phycobiliproteins which we have investigated so far, we have observed fast isotropic decays in the order of a few tens of picoseconds and in most cases also fast riseterms (Holzwarth et al., 1983b; Wehrmeyer et al., 1985; Holzwarth, 1985). This finding is in line with the results from other groups (Hanzlik et al., 1985; Kobayashi et al., 1979) and also with the observation of fast anisotropy decays (Hefferle et al., 1983a,b; Hefferle et al., 1984a, b). Different results have been reported recently for isolated R-phycoerythrin and allophycocyanin (Glazer et al., 1985). Both of these phycobiliproteins have not been studied by us so far, however.

While the fast decay components $\left(\tau_{1}=22\right.$ and 36 ps) of the two preparations (with and without linker peptides) can be clearly interpreted in terms of intramolecular energy transfer, the situation is more difficult for the $\tau_{2}$ components ( 200 and $120 \mathrm{ps}$ ). Their contribution to the total amplitude is relatively small. They might nevertheless also originate from energy transfer processes. One possibility would be energy transfer from pigments with somewhat distorted apoproteins and/or prosthetic groups. Both of these short decay components show variations with the two preparations which may be significant with respect to the functions of the colorless linker peptides. The function of these colorless polypeptides is, however, presently less well understood for $M$. laminosus than for other species (Glazer et al., 1983). Both $\tau_{1}$ and $\tau_{2}$ are reduced in the preparation containing these peptides. This effect may indicate an increased coupling between $\mathrm{s}$ and $\mathrm{f}$-chromophores induced by the linker peptides.

\section{The long-lived decay components}

We observe in each of the two preparations two long-lived decay components with lifetimes in the range of $\tau_{3} \approx 800 \mathrm{ps}$ and $\tau_{4} \approx 1450 \mathrm{ps}$. Both of these decays have very similar amplitudes (see Table 1). The long-lived decay is attributed to the emission from the f-chromophores and one would a priori expect a single lifetime. The two decays of about equal amplitude indicate a basic heterogeneity in these pigments. Heterogeneous chromophore populations have already been inferred for integral C-phycocyanin (Switalski and Sauer, 1984) and for isolated subunits (Switalski and Sauer, 1984; Hefferle et al., 1984a) from the observation of two long-lived fluorescence components. The longest lifetime reported by Switalski and Sauer (1984) for integral C-phycocyanin from Anabaena variabilis was similar to the $\tau_{4}$ component while the other was substantially shorter. These differences can be explained readily by the analysis in terms of two
(Switalski and Sauer, 1984) and four exponentials (this work). Otherwise our results are in good agreement with theirs and confirm the heterogeneity in the f-chromophores. It is interesting to note that, using different isolation procedures and different organisms, very similar results are obtained with respect to this heterogeneity. Apparently it is not caused by a modification of the proteins introduced during the isolation procedures but might rather be related to the aggregation state, the specific interaction with linker peptides and/or variations in the chromophore geometries. In fact a fluorescence study of C-phycocyanin from Synechococcus 6301 indicates a much less pronounced heterogeneity in the hexameric aggregation state (Holzwarth, 1985). The $\tau_{2}$ components of 200 and $120 \mathrm{ps}$ observed here could perhaps be explained in terms of this heterogeneity. Our data suggest that the linker peptides and aggregation state play a more pronounced role than do any species dependent differences (Seibert and Connolly, 1984). Since C-phycocyanin contains two 's'- and one 'f'-type chromophore, another possible explanation for this second fast component would be energy transfer from the second ' $s$ 'chromophore. This point deserves further investigations.

\section{CONCLUSIONS}

The fluorescence decays of trimeric $\mathrm{C}$-phycocyanin show a complex pattern of rise- and decay terms which requires a four-exponential model function. For the analysis of this complex fluorescence kinetics the simultaneous data analysis procedure turned out to be of great importance. The very fast decay and rise components found in this study can be rationalized by intramolecular energy transfer. Given the more limited time-resolution and/or componentresolution of previous time-resolved studies of the excited state kinetics of $\mathrm{C}$-phycocyanin, our data are in agreement with these results. Differences seem to be present in the fast energy transfer kinetics between the preparations with or without additional colorless peptides. These differences may be relevant with respect to the function of the linker peptides as modulators for the chromophore conformation and/ or chromophore/- protein interaction in phycobiliproteins.

Acknowledgements-We thank Dr. R. Hensel for the analytical ultracentrifuge measurements. This work has been supported in part by the Deutsche Forschungsgemeinschaft. We also thank Professors K. Schaffner and W. Rüdiger for continuing interest and support of this work.

\section{REFERENCES}

Binder, A., K. Wilson and H. Zuber (1972) Cphycocyanin from the thermophilic blue-green alga Mastigocladus laminosus. Isolation, characterization and subunit composition. FEBS Lett. 20, 111-116.

Byfield, P. G. H. and H. Zuber (1972) Chromophorecontaining peptide sequences in C-phycocyanin from 
Mastigocladus laminosus. Fed. Eur. Biochem. Soc. 28 , $36-40$.

Gantt, E. (1980) Structure and function of phycobilisomes: Light harvesting pigment complexes in red and blue-green algae. Int. Rev. Cytol. 66, 45-80.

Gantt, E. (1981) Phycobilisomes. Annu. Rev. Plant Physiol. 32, 327-347.

Gillbro, T., A. Sandstrom, V. Sundstrom and A. R. Holzwarth (1983) Polarized absorption picosecond kinetics as a probe of energy transfer in phycobilisomes of Synechococcus 6301. FEBS Lett. 162, 64-68.

Gillbro, T., A. Sandstrom, V. Sundstrom, J. Wendler and A. R. Holzwarth (1985) Picosecond study of energy transfer kinetics in phycobilisomes of Synechococcus 6301 and the mutant AN 112. Biochim. Biophys. Acta $808,52-65$.

Glazer, A. N. (1983) Comparative biochemistry of photosynthetic light-harvesting systems. Annu. Rev. Biochem. 52, 125-127.

Glazer, A. N., S. Fang and D. M. Brown (1973) Spectroscopic properties of C-phycocyanin and of its alpha and beta subunits. J. Biol. Chem. 248, 5679-5685.

Glazer, A. N., D. J. Lundell, G. Yamanaka and R. C. Williams (1983) The structure of a simple phycobilisome. Ann. Microbiol. 134B, 159-180.

Glazer, A. N., S. W. Yeh, S. P. Webb and J. H. Clark (1985) Disk-to-disk transfer as the rate-limiting step for energy flow in phycobilisomes. Science 227, 419-423.

Hanzlick, C. A., L. E. Hancock, R. S. Knox, D. GuardFriar and R. MacColl (1985) Picosecond fluorescence spectroscopy of the biliprotein phycocyanin 612. Direct evidence for fast energy transfer. $J$. Luminesc. 34, 99-106.

Hefferle, P., P. Geiselhart, T. Mindl, S. Schneider, W. John and H. Scheer (1984a) Time-resolved polarized fluorescence of C-phycocyanin and its subunits from Mastigocladus laminosus. Z. Naturforsch. 39c, 606-616.

Hefferle, P., W. John, H. Scheer and S. Schneider (1984b) Thermal denaturation of monomeric and trimeric phycocyanins studied by static and polarized time-resolved fluorescence spectroscopy. Photochem. Photobiol. 39, 221-232.

Hefferle, P., M. Nies, W. Wehrmeyer and S. Schneider (1983a) Picosecond time-resolved fluorescence study of the antenna system isolated from Mastigocladus laminosus Cohn. II. Constituent biliproteins in various forms of aggregation. Photobiochem. Photobiophys. 5, 325-334.

Hefferle, P., M. Nies, W. Wehrmeyer and S. Schneider (1983b) Picosecond time-resolved fluorescence study of the antenna system isolated from Mastigocladus laminosus Cohn. I. Functionally intact phycobilisomes. Photobiochem. Photobiophys. 5, 41-51.

Holzwarth, A. R. (1985) Energy transfer kinetics in phycobilisomes. In Antennas and Reaction Centers of Photosynthetic Bacteria, (Edited by M. E. MichelBeyerle), pp. 45-52. Springer-Verlag, Berlin.

Holzwarth, A. R., J. Wendler, K. Schaffner, V. Sundstrom, A. Sandstrom and T. Gillbro (1983a) Picosecond kinetics of excited state relaxation in biliverdin dimethyl ester. Isr. J. Chem. 23, 223-231.

Holzwarth, A. R., J. Wendler and W. Wehrmeyer (1982) Picosecond time resolved energy transfer in isolated phycobilisomes from Rhodella violacea (Rhodophyceae). Photochem. Photobiol. 36, 479-487.

Holzwarth, A. R., J. Wendler and W. Wehrmeyer (1983b) Studies on chromophore coupling in isolated phycobiliproteins. I. Picosecond fluorescence kinetics of energy transfer in phycocyanin 645 from Chroomonas sp. Biochim. Biophys. Acta 724, 388-395.

Karukstis, K. K. and K. Sauer (1983) Fluorescence decay kinetics of chlorophyll in photosynthetic membranes. J. Cell Biochern. 23, 131-158.
Knutson, J. R., J. M. Beechem and L. Brand (1983) Simultaneous analysis of multiple fluorescence decay curves: A global approach. Chem. Phys. Lett. 102. 501-507.

Kobayashi, T., E. O. Degenkolb, R. Bersohn, P. M. Rentzepis, R. MacColl and D. S. Berns (1979) Energy transfer among the chromophores in phycocyanins measured by picosecond kinetics. Biochemistry 18 , 5073-5078.

MacColl, R. and D. S. Berns (1981) Biliproteins: Some relationships among aggregation states, spectra, and excitation-energy transfer. Isr. J. Chem. 21, 296-300.

Marquardt, D. W. (1963) An algorithm for leastsquares estimation of nonlinear parameters. J. Soc. Ind. Appl. Math. 11, 431-441.

Mimuro, M., I. Yamazaki, T. Murao, K. Yoshihara and Y. Fujita (1984) Excitation energy transfer in phycobilin-chlorophyll $a$ system in blue-green and red algae. $A d v$. Photosyn. Res. 1, 21-28.

Mimuro, M., I. Yamazaki, T. Yamazaki and Y. Fujita (1985) Excitation energy transfer from phycobilisome to chlorophyll $a$ in the in vivo system of blue-green algae: $A$ problem on the role of the 680 nm-emitting allophycocyanin. Photochem. Photobiol. 41, 597-603.

Pellegrino, F., D. Wong, R. R. Alfano and B. A Zilinskas (1981) Fluorescence relaxation kinetics and quantum yield from the phycobilisomes of the blue-green alga Nostoc sp. measured as a function of single picosecond pulse intensity. Photochem. Photobiol. 34, 691-696.

Porter, G., C. J. Tredwell, G. F. W. Searle and J. Barber (1978) Picosecond time-resolved energy transfer in Porphyridium cruentum. Part I. In the intact alga. Biochim. Biophys. Acta 501, 232-245.

Scheer, H. (1982) Phycobiliproteins: Molecular aspects of a photosynthetic anterna system. In Mol. Biol. Biochem. Biophys. 35: Light Reaction Path of Photosynthesis (Edited by F. K. Fong), pp. 7-45. SpringerVerlag, Berlin.

Scheer, H. (1986) Excitation transfer in phycobiliproteins. In Encyclopedia of Plant Physiology: Photosynthesis III (Edited by Staehelin and Arntzen), Vol. 19. In press. Springer-Verlag, Berlin.

Schirmer, T., W. Bode, R. Huber, W. Sidler and H. Zuber (1985a) The crystal and molecular structure of C-phycocyanin from Mastigocladus laminosus and its implications for function and evolution. In Optical Properties and Structure of Tetrapyrroles (Edited by G. Blauer and $H$. Sund), pp. 446-449. Walter de Gruyter, Berlin.

Schirmer, T., W. Bode, R. Huber, W. Sidler and H. Zuber (1985b) X-ray crystallographic structure of the light-harvesting biliprotein C-phycocyanin from the thermophilic cyanobacterium Mastigocladus laminosus and its resemblance to globin structures. J. Mol. Biol. 184, 257-277.

Searle, G. F. W., J. Barber, G. Porter and C. J. Tredwell (1978) Picosecond time-resolved energy transfer in Porphyridium cruentum. Part II. In the isolated light harvesting complex (phycobilisomes). Biochim. Biophys. Acta 501, 246-256.

Seibert, M. and J. C. Connolly (1984) Fluorescence properties of c-phycocyanin isolated from a thermophilic cyanobacterium. Photochem. Photobiol. 40, 267-271.

Suter, G. W., P. Mazzola, J. Wendler and A. R. Holzwarth (1984) Fluorescence decay kinetics in phycobilisomes from the blue-green alga Synechococcus 6301. Biochim. Biophys. Acta 766, 269-276.

Switalski, S. C. and K. Sauer (1984) Energy transfer among the chromophores of C-phycocyanin from Anabaena variabilis using steady state and time-resolved fluorescence spectroscopy. Photochem. Photobiol. 40, 423-427. 
Teale, F. W. J. and R. E. Dale (1970) Isolation and spectral characterization of phycobiliproteins. Biochem. J. 116, 161-169.

Wehrmeyer, W. (1983a) Organization and composition of cyanobacterial and rhodophycean phycobilisomes. In Photosynthetic Prokaryotes (Edited by G. C. Papageorgiou and L. Packer), pp. 1-22. Elsevier, Amsterdam.

Wehrmeyer, W. (1983b) Phycobiliproteins and phycobiliprotein organization in the photosynthetic apparatus of cyanobacteria, red algae and cryptophytes. In Proteins and Nucleic Acids in Plant Systematics (Edited by U. Jensen and D. E. Fairbrothers), pp. 143-167. SpringerVerlag, Berlin.

Wehrmeyer, W., J. Wendler and A. R. Holzwarth (1985) Biochemical and functional characterization of a peripheral unit of the phycobilisomes from Porphyridium cruentum. Measurement of picosecond energy transfer kinetics. Eur. J. Cell Biol. 36, 17-23.

Wong, D., F. Pellegrino, R. R. Alfano and B. A. Zilinskas (1981) Fluorescence relaxation kinetics and quantum yield from the isolated phycobiliproteins of the bluc-green alga Nostoc $\mathrm{sp}$. measured as a function of single picosecond pulse intensity. I. Photochem. Photobiol. 33, 651-662.

Yamazaki, I., M. Mimuro, T. Murao, T. Yamazaki, K. Yoshihara and Y. Fujita (1984) Excitation energy transfer in the light harvesting antenna system of the red alga Porphyridium cruentum and the blue-green alga Anacystis nidulans: Analysis of time-resolved fluorescence spectra. Photochem. Photobiol. 39, 233-240. 